\title{
Multi-sensor Data Fusion Based on Belief Functions and Possibility Theory: Close Range Antipersonnel Mine Detection and Remote Sensing Mined Area Reduction
}

\author{
Nada Milisavljević1, Isabelle Bloch ${ }^{2}$ and Marc Acheroy ${ }^{1}$ \\ ${ }^{1}$ Signal and Image Centre, Royal Military Academy \\ Belgium \\ ${ }^{2}$ TSI department, Ecole Nationale Supérieure des Télécommunications
}

France

\section{Introduction}

Two main humanitarian mine action types may benefit from multi-sensor data fusion techniques: 1) close range antipersonnel (AP) mine detection and 2) mined area reduction. Data fusion for these two applications is presented here. Close range detection consists of detection of (sub-)surface anomalies that may be related to the presence of mines (e.g., detection of metals using a metal detector, or detection of temperature differences using an infrared camera) and/or detection of explosive materials. Area reduction consists in identifying the mine-free areas out of the mine-suspected areas.

For both close range detection and area reduction, efficient modeling and fusion of extracted features can improve the reliability and quality of single-sensor based processing (Acheroy, 2003). However, due to a huge variety of scenarios and conditions within a minefield (specific moisture, depth, burial angles) and between different minefields (types of mines, types of soil, minefield structure), a satisfactory performance of humanitarian mine action tools can only be obtained using multi-sensor and data fusion approaches (Keller et al., 2002; Milisavljević \& Bloch, 2005). Furthermore, as the sensors used are typically detectors of \& different anomalies, combinations of these complementary pieces of information may improve the detection and classification results. Finally, in order to take into account the inter- and intra-minefield variability, uncertainty, ambiguity and partial knowledge, fuzzy set or possibility theory (Dubois \& Prade, 1980) as well as belief functions (Smets, 1990b) within the framework of the Dempster-Shafer theory (Shafer, 1976) prove to be useful.

\ In case of close range detection, a detailed analysis of modeling and fusion of extracted features is presented and two fusion methods are discussed, one based on the belief functions and the other based on the possibility theory. They are illustrated using real data coming from three complementary sensors (metal detector, ground-penetrating radar and infrared camera), gathered within the Dutch project HOM-2000 (de Yong et al., 1999). These 
results are obtained within two Belgian humanitarian demining projects, HUDEM and BEMAT. For mined area reduction, three approaches are shown, two of them based on the belief functions and one based on the fuzzy logic. They are also illustrated using real data of synthetic-aperture radar and multi-spectral sensors, collected within the EU project on space and airborne mined area reduction tools (SMART). In all cases, importance of collateral information (knowledge about types of mines, mine records, etc.) is demonstrated.

\section{About Mine Detection and Mined Area Reduction}

\subsection{Close Range AP Mine Detection}

Due to the high variety of types of mines and of conditions in which mines can be found, there is no single sensor used for humanitarian mine detection that can reach the necessarily high detection rate in all possible scenarios. Therefore, a way towards finding a solution is in taking the best from several complementary sensors. One of the most promising sensor combinations consists in an infrared camera (IR), an imaging metal detector (MD) and a ground-penetrating radar (GPR). We present here two approaches for combining these sensors, which can be easily adapted for other sensors and their combinations. These approaches are based on the belief function theory and on the possibility theory.

Most of the work done in the field of fusion of dissimilar mine detection sensors is based on statistical approaches (Cremer et al., 2001; Yee, 2001). Examples of alternative approaches are (Stanley et al., 2002) (neural networks) and (Auephanwiriyakul et al., 2002) (fuzzy fusion of classifiers). The statistical approaches lead to good results for a particular scenario, but they ignore or just briefly mention that several important problems have to be faced in this domain of application (Milisavljević \& Acheroy, 1999), once more general solutions are looked for. Namely, the data are highly variable depending on the context and conditions. Besides, the data are not numerous enough to allow for a reliable statistical learning, and they do not give precise information on the type of mine (ambiguity between several types). Finally, it is not possible to model every object (neither mines nor objects that could be confused with them). In addition, a number of the fusion attempts in this domain of application, e.g. (den Breejen et al., 1999; Perrin, 2001), treat every alarm as a mine, and not as an object that could be a mine, but a false alarm as well.

In a previous work (Milisavljević \& Bloch, 2003), a method based on the belief functions (Shafer, 1976; Smets \& Kennes, 1994; Smets, 1994) has been proposed. In this chapter, we compare it with an alternative approach, based on the possibility theory, in order to take advantage of the flexibility in the choice of combination operators (Dubois \& Prade, 1985). This is exploited here to account for the different characteristics of the sensors to be combined. To our knowledge, in the domain of humanitarian mine detection, there is no attempt to apply the two fusion theories in parallel or to compare them. In other domains, there are some works that compare the two approaches, such as (Dubois et al., 2001), where the belief function theory is opposed to the qualitative possibility theory and illustrated on a fictitious example of the assessment of the value of a candidate. On the contrary to that paper, we apply the quantitative possibility theory here.

\subsection{Remote Sensing for Mined Area Reduction}

AP mines affect at least 84 countries and 8 areas not internationally recognized as independent states (ICBL, 2005). Thanks to the Mine Ban Treaty, mine clearing operations 
have been organized in a more controlled and effective way, yet mine clearance remains a slow and resource demanding process. It is estimated that, on average, a deminer clears 10 $\mathrm{m}^{2}$ during a working day using conventional tools such as metal detectors and prodders. Thus, humanitarian mine clearance operations must be understood and designed correctly, providing efficient aid to innocent people who may be severely injured by this dreadful pollution. The recommendations made during the Standing Committee on Mine Clearance, Mine Risk Education and Mine Action Technologies state that: 1) technologists should avoid building technologies based on assumed needs and should work interactively with endusers, 2) appropriate technologies could save human lives and increase mine action efficiency, and 3) nothing is more important than understanding the working environment (Acheroy, 2003; JMU). Besides the very long time needed to clear polluted terrain, actual demining campaigns show that the false alarm rate is very large, the threat of plastic mines (which cannot be detected by metal detectors) is not negligible and the variety of mine clearance scenarios is high, depending on the country, the region, the climate etc. These facts prove that the humanitarian mine detection is a very complex problem. In addition, the experience shows that it will be a long process to achieve a mine-free world, so the concept of a mine-free world is evolving softly toward the concept of a mine impact-free world, although a mine-free world remains the final goal of the Mine Ban Treaty. By this, the first priority of mine action becomes in allowing affected regions to reach their level of socioeconomic standards. This new vision increases the importance of tools that facilitate prioritization and contribute to a rational and efficient distribution of the available resources. Several information management systems are developed and used. An example is the Information Management System for Mine Action - IMSMA (IMSMA, 1999), developed thanks to the Geneva International Centre for Humanitarian Demining (GICHD) and in use in more than 40 affected countries. Other examples are systems completing IMSMA, such as the EODIS system (Askelin, 1999) developed by SWEDEC in Sweden and the PARADIS system (Delhay et al., 2005) developed by the Royal Military Academy (RMA) in Belgium. Possible entries of such management systems are danger and risk assessment maps provided by the Space and airborne Mined Area Reduction Tools (SMART) project (SMART consortium, 2004; Acheroy, 2005), funded by the European Commission. The maps, obtained using data fusion, synthesize the knowledge gathered from the existing data. In the framework of SMART, the fusion module, detailed in this chapter, is a very important step, since it allows for taking the best from all available data, and of the large efforts made in the scientific community to design detectors and classifiers adapted to these data. It has proven to be a required step before constructing risk maps. This is an improvement in comparison to existing information management systems in this area. In particular, the proposed approach exploits all available data and knowledge and automatically adapts to the quality of the detectors and classifier outputs.

\section{Belief Functions and Possibility Theory for Numerical Information Fusion}

\subsection{Belief Function Fusion - Overview}

Belief function theory or Dempster-Shafer evidence theory (DS) (Shafer, 1976; Smets, 1990b) allows representing both imprecision and uncertainty, using plausibility and belief functions derived from a mass function. The mass of a proposition $A$ is a part of the initial unitary amount of belief that supports that the solution is exactly in $A$. It is defined as a 
function $m$ from $2^{\Theta}$ into $[0,1]$, where $\Theta$ is the decision space, also called frame of discernment or full set. Usually the following constraints are imposed:

$$
\begin{gathered}
m(\varnothing)=0, \\
\sum_{A \subseteq \Theta} m(A)=1 .
\end{gathered}
$$

In this formalism, any combination of possible decisions from the decision space can be quantified rather than considering only the singletons of $\Theta$. This is one of the main advantages of the DS approach. Indeed, it leads to a very flexible and rich modeling, able to fit a very large class of situations, occurring in image fusion in particular. Examples of situations where DS theory may be successfully used include ignorance or partial ignorance, confusion between classes (in one or several sources of information), partial reliability, etc (van Cleynenbreugel et al., 1991; Mascle et al., 1997; Le Hégarat-Mascle et al., 1998; Tupin et al., 1999; Milisavljević \& Bloch, 2003; Milisavljević \& Bloch, 2005).

In the DS framework, masses assigned by different sources (e.g., classifiers) are combined by the orthogonal rule of Dempster (Shafer, 1976):

$$
m_{i j}(S)=\sum_{\substack{k, l \\ A_{k} \cap B_{l}=S}} m_{i}\left(A_{k}\right) \cdot m_{j}\left(B_{l}\right)
$$

where $\mathrm{S}$ is any subset of the full set, while $m_{i}$ and $m_{j}$ are masses assigned by measures $i$ and $j$, and their focal elements are $A_{1}, A_{2}, \ldots, A_{p}$ and $B_{1}, B_{2}, \ldots, B_{q}$, respectively.

Dempster's rule is commutative and associative, meaning that it can be applied repeatedly, until all measures are combined, and that the result does not depend on the order used in the combination. After the combination in this unnormalized form (Smets, 1993), the mass that is assigned to the empty set:

$$
m_{i j}(\varnothing)=\sum_{\substack{k, l \\ A_{k} \cap B_{l}=\varnothing}} m_{i}\left(A_{k}\right) \cdot m_{j}\left(B_{l}\right)
$$

can be interpreted as a measure of conflict between the sources. It can be directly taken into account in the combination as a normalization factor. It is very important to consider this value for evaluating the quality of the combination: when it is high (in case of strong conflict), the normalized combination may not make sense and can lead to questionable decisions (Dubois \& Prade, 1988). Several authors suggest not normalizing the combination result (e.g., Smets, 1993), which corresponds to Eq. 3.

This fusion operator has a conjunctive behavior. This means that all imprecision on the data has to be introduced explicitly at the modeling level, in particular in the choice of the focal elements. For instance, ambiguity between two classes in one source of information has to be modeled using a disjunction of hypotheses, so that conflict with other sources can be limited and ambiguity can be possibly solved during the combination.

From a mass function, we can derive a belief function:

$$
\forall A \in 2^{\Theta}, \operatorname{Bel}(A)=\sum_{B \subseteq A, B \neq \varnothing} m(B)
$$

as well as a plausibility function:

$$
\forall A \in 2^{\Theta}, \operatorname{Pls}(A)=\sum_{B \cap A \neq \varnothing} m(B) .
$$

After the combination, the final decision is usually taken in favor of a simple hypothesis using one of several rules (Denœux, 1995): e.g. the maximum of plausibility (generally over simple hypotheses), the maximum of belief, the pignistic decision rule (Smets, 1990a), etc. 
For some applications, such as humanitarian demining, it may be necessary to give more importance to some classes (e.g., mines, since they must not be missed) at the decision level. Then maximum of plausibility can be used for the classes that should not be missed, and maximum of belief for the others (Milisavljević \& Bloch, 2001), as shown in Subsection 4.4.

\subsection{Fuzzy and Possibilistic Fusion - Overview}

In the framework of fuzzy sets and possibility theory (Zadeh, 1965, Dubois \& Prade, 1980), the modeling step consists in defining a membership function to each class or hypothesis in each source, or a possibility distribution over the set of hypotheses in each source.

Such models explicitly represent imprecision in the information, as well as possible ambiguity between classes or decisions.

For the combination step in the fusion process, the advantages of fuzzy sets and possibilities rely in the variety of combination operators, which may deal with heterogeneous information (Dubois \& Prade, 1985; Yager, 1991). Among the main operators, we find tnorms, t-conorms, mean operators, symmetrical sums, and operators taking into account conflict between sources or reliability of the sources. We do not detail all operators in this chapter, but they can be easily found in the literature, with a synthesis in (Bloch, 1996).

We classify these operators with respect to their behavior (in terms of conjunctive, disjunctive, compromise (Dubois \& Prade, 1985)), the possible control of this behavior, their properties and their decisiveness, which proved to be useful for several applications (Bloch, 1996). It should be noted that, unlike other data fusion theories (e.g., Bayesian or DempsterShafer combination), fuzzy sets provide a great flexibility in the choice of the operator, that can be adapted to any situation at hand. In particular nothing prevents using different operators for different hypotheses or different sources of information.

An advantage of this approach is that it is able to combine heterogeneous information, which is usually the case in multi-source fusion (as in both examples developed in the next sections), and to avoid to define a more or less arbitrary and questionable metric between pieces of information issued from these images, since each piece of information is converted in membership functions or possibility distributions over the same decision space.

Decision is usually taken from the maximum of membership or possibility values after the combination step. Constraints can be added to this decision, typically for checking for the reliability of the decision (is the obtained value high enough?) or for the discrimination power of the fusion (is the difference between the two highest values high enough?). Local spatial context can be used to reinforce or modify decisions (see the example in Section 5).

\section{Close-range Mine Detection}

\subsection{Measures}

From the data gathered by the sensors, a number of measures are extracted (Milisavljević \& Bloch, 2003) and modeled using the two approaches. These measures concern:

- $\quad$ the area and the shape (elongation and ellipse fitting) of the object observed using the IR sensor,

- the size of the metallic area in MD data,

- the propagation velocity (thus the type of material), the burial depth of the object observed using GPR, and the ratio between object size and its scattering function. 
Although the semantics are different, similar information can be modeled in both possibilistic and belief function models. The idea here is to design the possibility and mass functions as similarly as possible and to concentrate on the comparison at the combination step.

The main difference relies in the modeling of ambiguity. The semantics of possibility leads to model ambiguity between two hypotheses with the same degrees of possibilities for these two hypotheses (e.g., Eq. 7 and Eq. 12). On the contrary, the reasoning on the power set of hypotheses in the belief function theory leads to assigning a mass to the union of these two hypotheses (e.g., Eq. 9 and Eq. 14).

Another distinction concerns the ignorance. It is explicitly modeled in the belief function theory, through a mass on the whole set (to guarantee the normalization of the mass function over the power set), while it is only expressed implicitly in the possibilistic model, through the absence of normalization constraint.

IR measures: The possibility degrees derived from elongation and ellipse fitting measures are represented by $\pi_{1 I}$ and $\pi_{2 I}$, respectively. Being related to shape regularity, they are defined for a regular-shaped mine $(M R)$, an irregular-shaped mine $(M I)$, a regular-shaped non-dangerous (i.e., friendly) object $(F R)$ and an irregularly shaped friendly object $(F I)$.

In the belief function framework, the full set is: $\Theta=\{M R, M I, F R, F I\}$. As elongation and ellipse fitting aim at distinguishing regular and irregular shapes, masses assigned by these two measures, $m_{1 I}$ and $m_{2 I}$, are split between $M R \cup F R, M I \cup F I$ and $\Theta$.

Regarding elongation, we calculate $r_{1}$ as the ratio between minimum and maximum distance of bordering pixels from the center of gravity (we work on thresholded images) and $r_{2}$ as the ratio of minor and major axis obtained from second moment calculation. Using these two ratios, the following possibility degrees are derived:

$$
\begin{aligned}
& \pi_{1 I}(M R)=\pi_{1 I}(F R)=\min \left(r_{1}, r_{2}\right), \\
& \pi_{1 I}(M I)=\pi_{1 I}(F I)=1-\pi_{1 I}(M R) .
\end{aligned}
$$

In the framework of belief functions, for this measure, masses are defined as follows:

$$
\begin{gathered}
m_{1 I}(M R \cup F R)=\min \left(r_{1}, r_{2}\right), \\
m_{1 I}(M I \cup F I)=\left|r_{1}-r_{2}\right|,
\end{gathered}
$$

and the full set takes the rest:

$$
m_{1 I}(\Theta)=1-\max \left(r_{1}, r_{2}\right)
$$

In case of ellipse fitting, let $A_{o e}$ be the part of object area that belongs to the fitted ellipse as well, $A_{o}$ the object area, and $A_{e}$ the ellipse area. Then we define:

$$
\begin{gathered}
\pi_{2 I}(M R)=\pi_{2 I}(F R)=\max \left(0, \min \left\{\frac{A_{o e}-5}{A_{o}}, \frac{A_{o e}-5}{A_{e}}\right\}\right), \\
\pi_{2 I}(M I)=\pi_{2 I}(F I)=1-\pi_{2 I}(M R) .
\end{gathered}
$$

Masses for this measure are the following ones:

$$
\begin{gathered}
m_{2 I}(M R \cup F R)=\max \left(0, \min \left\{\frac{A_{o e}-5}{A_{o}}, \frac{A_{o e}-5}{A_{e}}\right\}\right), \\
m_{2 I}(M I \cup F I)=\max \left\{\frac{A_{e}-A_{o e}}{A_{e}}, \frac{A_{o}-A_{o e}}{A_{o}}\right\},
\end{gathered}
$$




$$
m_{2 I}(\Theta)=1-m_{2 I}(M R \cup F R)-m_{2 I}(M I \cup F I) .
$$

Note that in cases where it is sure that all mines have a regular shape, the possibility degrees of $M R$ can be reassigned to mines of any shape $(M=M R \cup M I)$ while the possibility degrees of $M I$ can be reassigned to friendly objects of any shape $(F=F R \cup F I)$. Similarly, masses given to $M R \cup F R$ can be reassigned to $M$, while masses given to $M I \cup F I$ can be reassigned to $F$.

The area directly provides a degree $\pi_{3 I}(M)$ of being a mine. Namely, since the range of possible AP mine sizes is approximately known, the degree of possibility of being a mine is derived as a function of the measured size:

$$
\pi_{3 I}(M)=\frac{a_{I}}{a_{I}+0.1 \cdot a_{\operatorname{Im} \text { in }}} \cdot \exp \frac{-\left[a_{I}-0.5 \cdot\left(a_{\operatorname{Im} i n}+a_{\operatorname{Imax}}\right)\right]^{2}}{0.5 \cdot\left(a_{\operatorname{Im} a x}-a_{\operatorname{Im} \text { in }}\right)^{2}},
$$

where $a_{I}$ is the actual object area on the IR image, while the approximate range of expectable mine areas is between $a_{I \min }$ and $a_{I \max }$ (for AP mines, it is reasonable to set $a_{\text {Imin }}=15 \mathrm{~cm}^{2}$ and $a_{\text {Imax }}=225 \mathrm{~cm}^{2}$ ). On the contrary, friendly objects can be of any size, so the possibility degree is set to one whatever the value of the size:

$$
\pi_{3 I}(F)=1
$$

The area/size mass assignment based on the above reasoning is given by:

$$
\begin{aligned}
m_{3 I}(\Theta)= & \frac{a_{I}}{a_{I}+0.1 \cdot a_{\mathrm{Im} i n}} \cdot \exp \frac{-\left[a_{I}-0.5 \cdot\left(a_{\operatorname{Im} i n}+a_{\operatorname{Im} a x}\right)\right]^{2}}{0.5 \cdot\left(a_{\operatorname{Im} a x}-a_{\operatorname{Im} i n}\right)^{2}}, \\
& m_{3 I}(F R \cup F I)=1-m_{3 I}(\Theta) .
\end{aligned}
$$

MD measures: In reality, MD data are usually saturated and data gathering resolution in the cross-scanning direction is typically very poor, so the MD information used consists of only one measure, which is the width of the region in the scanning direction, $w[\mathrm{~cm}]$. As friendly objects can contain metal of any size, we define:

$$
\pi_{M D}(F)=1 \text {. }
$$

If there is some knowledge on the expected sizes of metal in mines (for AP mines, this range is typically between $5 \mathrm{~cm}$ and $15 \mathrm{~cm}$ ), we can assign possibilities to mines as, e.g.:

$$
\pi_{M D}(M)=\frac{w}{20} \cdot[1-\exp (-0.2 \cdot w)] \cdot \exp \left(1-\frac{w}{20}\right) .
$$

The corresponding mass functions are:

$$
\begin{gathered}
m_{M D}(\Theta)=\frac{w}{20} \cdot[1-\exp (-0.2 \cdot w)] \cdot \exp \left(1-\frac{w}{20}\right), \\
m_{M D}(F R \cup F I)=1-m_{M D}(\Theta) .
\end{gathered}
$$

GPR measures: All three GPR measures provide information about mines.

In case of burial depth information $(D)$, friendly objects can be found at any depth, while it is known that there is some maximum depth up to which AP mines can be expected, mainly due to their activation principles. However, due to soil perturbations, erosions etc., mines can, by time, go deeper or shallower than the depth at which they were initially buried. In any case, they can rarely be found buried below $25 \mathrm{~cm}\left(D_{\max }\right)$. Thus, for this GPR measure, possibility distributions $\pi_{1 G}$ for mines and friendly object can be modeled as follows:

$$
\begin{gathered}
\pi_{1 G}(M)=\frac{1}{\cosh \left(D / D_{\max }\right)^{2}}, \\
\pi_{1 G}(F)=1 .
\end{gathered}
$$


In terms of belief functions, the masses for this measure are:

$$
\begin{aligned}
& m_{1 G}(\Theta)=\frac{1}{\cosh \left(D / D_{\max }\right)^{2}}, \\
& m_{1 G}(F R \cup F I)=1-m_{1 G}(\Theta) .
\end{aligned}
$$

Another GPR measure exploited here is the ratio between object size and its scattering function, $d / k$. Again, friendly objects can have any value of this measure, while for mines, there is a range of values that mines can have, and outside that range, the object is quite certainly not a mine:

$$
\begin{gathered}
\pi_{2 G}(M)=\exp \left(-\frac{\left[(d / k)-m_{d}\right]^{2}}{2 \cdot p^{2}}\right), \\
\pi_{2 G}(F)=1,
\end{gathered}
$$

where $m_{d}$ is the $d / k$ value at which the possibility distribution reaches its maximum value (here, $m_{d}=700$, chosen based on prior information), and $p$ is the width of the exponential function (here, $p=400$ ).

Similarly, the mass assignments for this measure are:

$$
\begin{gathered}
m_{2 G}(\Theta)=\exp \left(-\frac{\left[(d / k)-m_{d}\right]^{2}}{2 \cdot p^{2}}\right), \\
m_{2 G}(F R \cup F I)=1-m_{2 G}(\Theta) .
\end{gathered}
$$

Finally, propagation velocity, $v$, can provide information about object identity. Here, we extract depth information on a different way than in the case of the burial depth measure (Milisavljević et al., 2003) and we preserve the sign of the extracted depth. This information indicates whether a potential object is above the surface. If that is the case, the extracted $v$ should be close to $c=3 \cdot 10^{8} \mathrm{~m} / \mathrm{s}$, the propagation velocity in vacuum. Otherwise, if the sign indicates that the object is below the soil surface, the value of $v$ should be around the values for the corresponding medium, e.g., from $5.5 \cdot 10^{7} \mathrm{~m} / \mathrm{s}$ to $1.73 \cdot 10^{8} \mathrm{~m} / \mathrm{s}$ in case of sand:

$$
\pi_{3 G}(M)=\exp \left(-\frac{\left(v-v_{\max }\right)^{2}}{2 \cdot h^{2}}\right),
$$

where $v_{\max }$ is the value of velocity which is the most typical for the medium (here, for sand, it is $0.5 \cdot\left(5.5 \cdot 10^{7}+1.73 \cdot 10^{8}\right)=1.14 \cdot 10^{8} \mathrm{~m} / \mathrm{s}$, and for air, it is equal to $\left.c\right)$, and $h$ is the width of the exponential function (here, $h=6 \cdot 10^{7} \mathrm{~m} / \mathrm{s}$ ). Once again, friendly objects can have any value of the velocity:

$$
\pi_{3 G}(F)=1 \text {. }
$$

The corresponding mass functions are:

$$
\begin{gathered}
m_{3 G}(\Theta)=\exp \left(-\frac{\left(v-v_{\max }\right)^{2}}{2 \cdot h^{2}}\right), \\
m_{3 G}(F R \cup F I)=1-m_{3 G}(\Theta) .
\end{gathered}
$$

\subsection{Combination}

The combination of possibility degrees, as well as of masses, is performed in two steps. The first one applies to all measures derived from one sensor. The second one combines results obtained in the first step for all three sensors. 
In case of possibilities, only the combination rules related to mines are considered. The issue of combination rules for friendly objects is discussed in Subsection 4.4.

Let us first detail the first step for each sensor. For IR, since mines can be regular or irregular, the information about regularity on the level of each shape measure is combined using a disjunctive operator (here the max):

$$
\begin{aligned}
& \pi_{1 I M}=\max \left(\pi_{1 I}(M R), \pi_{1 I}(M I)\right), \\
& \pi_{2 I M}=\max \left(\pi_{2 I}(M R), \pi_{2 I}(M I)\right) .
\end{aligned}
$$

The choice of the maximum (smallest disjunction and idempotent operator) as a t-conorm is related to the fact that the measures cannot be considered as completely independent from each other. Thus, there is no reason to reinforce the measures by using a larger $\mathrm{t}$-conorm, and the idempotent one is preferable in such situations. These two shape constraints should be both satisfied to have a high degree of possibility of being a mine, so they are combined in a conjunctive way (using a product). Finally, the object is possibly a mine if it has a size in the expected range or if it satisfies the shape constraint, hence the final combination for IR is:

$$
\pi_{I}(M)=\pi_{3 I}(M)+\left[1-\pi_{3 I}(M)\right] \cdot \pi_{1 I M} \cdot \pi_{2 I M} \cdot
$$

The conjunction in the second term guarantees that $\pi_{I}(M)$ is in $[0,1]$.

In case of GPR, it is possible to have a mine if the object is at shallow depths and its dimensions resemble a mine and the extracted propagation velocity is appropriate for the medium. Thus, the combination of the obtained possibilities for mines is performed using a $\mathrm{t}$-norm, expressing the conjunction of all criteria. Here the product t-norm is used:

$$
\pi_{G}(M)=\pi_{1 G}(M) \cdot \pi_{2 G}(M) \cdot \pi_{3 G}(M) .
$$

For MD, as there is just one measure used, there is no first combination step and the possibility degrees obtained using Eqs. 21 and 22 are directly used.

In case of possibilities, the second combination step is performed using the algebraic sum:

$$
\begin{aligned}
\pi(M)= & \pi_{I}(M)+\pi_{M D}(M)+\pi_{G}(M)-\pi_{I}(M) \cdot \pi_{M D}(M)-\pi_{I}(M) \cdot \pi_{G}(M)- \\
& -\pi_{M D}(M) \cdot \pi_{G}(M)+\pi_{I}(M) \cdot \pi_{M D}(M) \cdot \pi_{G}(M)
\end{aligned}
$$

leading to a strong disjunction (Dubois \& Prade, 1985; Bloch, 1996), as the final possibility should be high if at least one sensor provides a high possibility, reflecting the fact that it is better to assign a friendly object to the mine class than to miss a mine.

In the belief function framework, for IR and GPR, masses assigned by the measures of each of the two sensors are combined by Dempster's rule in unnormalized form (Eq. 3). A general idea for using the unnormalized form of this rule instead of more usual, normalized form is to preserve conflict (Milisavljević \& Bloch, 2001), i.e., mass assigned to the empty set, Eq. 4. Here, a high degree of conflict would indicate that either there are several objects and the sensors, as detectors of different physical phenomena, do not provide information on the same object, or some sources of information are not completely reliable. Our main interest is in the possibility that sensors do not refer to the same object, as the unreliability can be modeled and resolved through discounting factors (Milisavljević \& Bloch, 2005). After combining masses per sensor, the fusion of sensors is performed, using Eq. 3 again. If the mass of the empty set after combination of sensors is high, they should be clustered as they do not sense the same object. 


\subsection{Comparison of the Combination Equations}

For IR, based on Eqs. 6-20 and 39, it can be shown that

$$
P l_{I}(M) \leq \pi_{I}(M) \text {. }
$$

This is in accordance with the least commitment principle used in the possibilistic model, as usually done in this framework.

As far as MD is concerned, there is no difference since it provides only one measure.

In case of GPR, based on the comparison of Eqs. 25 and 27, Eqs. 29 and 31, as well as Eqs. 33 and 35, we can conclude that Eq. 40 can be rewritten as:

$$
\pi_{G}(M)=m_{1 G}(\Theta) \cdot m_{2 G}(\Theta) m_{3 G}(\Theta) \text {. }
$$

Furthermore, the application of the Dempster's rule (Eq. 3) to the mass assignments of the three GPR measures results in the fused mass of the full set for this sensor:

$$
m_{G}(\Theta)=m_{1 G}(\Theta) \cdot m_{2 G}(\Theta) m_{3 G}(\Theta)
$$

which leads to:

$$
\pi_{G}(M)=m_{G}(\Theta) .
$$

This means that the ignorance is modeled as a mass on $\Theta$ in the belief function framework, while it privileges the class that should not be missed $(M)$ in the possibilistic framework (i.e., the ignorance will lead to safely decide in favor of mines).

\subsection{Decision}

As the final decision about the identity of the object should be left to the deminer not only because his life is in danger but also because of his experience, the fusion output is a suggested decision together with confidence degrees.

In case of possibilities, the final decision is obtained by thresholding the fusion result for $M$ and providing the corresponding possibility degree as the confidence degree. As almost all possibility degrees obtained at the fusion output are either very low or very high, the selected regions having very low values of $\pi(M)$ (below 0.1 ) are classified as $F$, and the ones with very high values (above 0.7 ) are classified as $M$. Only a few regions exist at which the resulting possibility degree for $M$ has an intermediary value and there, as mines must not be missed, the decision is $M$. In the following, this decision approach is referred to as dec1.

An alternative ( $d e c 2$ ) for the final decision making is to derive the combination rule for $F$ as well, compare the final values for $M$ and $F$ and derive an adequate decision rule. Due to operation principles of GPR and MD, the measures of these two sensors can only give information where mines are possibly not. As they are non-informative with respect to friendly objects, it is not useful to combine their possibility degrees for $F$. Thus, for deriving the final combination rule for $F, \pi(F)$, we can rely only on IR, i.e.:

$$
\pi(F)=\pi_{I}(F) \text {. }
$$

In case of IR, since friendly objects can be regular or irregular, we apply a disjunctive operator (the max) for each of the shape constraints. In order to be cautious when deciding $F$, we combine the two shape constraints and the area measure using a conjunctive operator. Taking into account Eq. 18, this reasoning results in:

$$
\pi(F)=\max \left(\pi_{1 I}\left(F_{R}\right), \pi_{1 I}\left(F_{I}\right)\right) \cdot \max \left(\pi_{2 I}\left(F_{R}\right), \pi_{2 I}\left(F_{I}\right)\right) .
$$

Thus, in this alternative way to derive decisions, in regions where IR gives an alarm, the decision rule chooses $M$ or $F$ depending on which one of the two has a higher possibility value, given by Eqs. 41 and 58, respectively. In other regions, at which IR does not give an 
alarm although at least one of the two other sensors gives an alarm, the decision is based on the fusion result for $M$, as in $d e c 1$.

In case of belief functions, as shown in (Milisavljević \& Bloch, 2003), usual decision rules based on beliefs, plausibilities (Shafer, 1976) and pignistic probabilities (Smets, 1990a) do not give useful results because there are no focal elements containing mines alone (Milisavljević \& Bloch, 2001). As a consequence, these usual decision rules would always favor friendly objects. The underlying reason is that the humanitarian demining sensors are anomaly detectors and not mine detectors. In such a sensitive application, no mistakes are allowed so in case of any ambiguity, much more importance should be given to mines. Because of that, in (Milisavljević \& Bloch, 2003), guesses $G(A)$ are defined, where $A \in\{M, F, \varnothing\}$ :

$$
\begin{gathered}
G(M)=\sum_{M \cap B \neq \varnothing} m(B), \\
G(F)=\sum_{B \subseteq F, B \neq \varnothing} m(B), \\
G(\varnothing)=m(\varnothing) .
\end{gathered}
$$

In other words, the guess value of a mine is the sum of masses of all the focal elements containing mines, regardless their shape, and the guess of a friendly object is the sum of masses of all the focal elements containing nothing else but friendly objects of any shape, meaning that the guesses are a cautious way to estimate confidence degrees.

As the output of the belief function fusion module, the three possible outputs ( $M, F$, conflict) are provided together with the guesses, for each of the sensors and for their combination. For GPR, the focal elements are only $F$ and $\Theta$, so guesses for this sensor become simply:

$$
\begin{aligned}
& G_{G}(M)=m_{G}(\Theta), \\
& G_{G}(F)=m_{G}(F) .
\end{aligned}
$$

From Eqs. 45 and 51, we conclude that for GPR, the possibility degree of a mine is equal to the guess of a mine:

$$
\pi_{G}(M)=G_{G}(M) .
$$

Furthermore, Eqs. 6 and 48 show that the guess of a mine is equal to its plausibility, while Eqs. 5 and 49 show that the guess of a friendly object is equal to its belief. This means that the relation given by Eq. 42 shows, actually, that for IR:

$$
G_{I}(M) \leq \pi_{I}(M) \text {. }
$$

\subsection{Results}

The proposed approach has been applied to a set of known objects, buried in sand, leading to 36 alarmed regions in total: 21 mines $(\mathrm{M}), 7$ placed false alarms (PF, friendly objects) and 8 false alarms caused by clutter (FN, with no object).

The results of the possibilistic fusion are very promising, since all mines are classified correctly with the proposed approach, as can be seen in Table 1 . The numbers given in the parenthesis indicate the number of regions selected in the preprocessing step for further analysis, i.e. measure extraction and classification. Regarding the combination operators, the results given in this table are based on the combination proposed in Subsection 4.2 (Eqs. 3941). The second fusion step is important, since a decision taken after the first step provides only 18 mines for IR, 9 for MD and 13 for GPR. This illustrates the interest of combining heterogeneous sensors. 


\begin{tabular}{|c|c|c|c|c|c|}
\hline \multirow{2}{*}{$\begin{array}{c}\text { Classified correctly, possibility } \\
\text { theory }\end{array}$} & \multicolumn{3}{|c|}{ Sensors } & \multicolumn{2}{c|}{ Fusion } \\
\cline { 2 - 6 } & IR & MD & GPR & $\operatorname{dec} 1$ & $\operatorname{dec} 2$ \\
\hline M & 18 & 9 & 13 & 21 & 21 \\
(total: 21$)$ & $(18)$ & $(9)$ & $(13)$ & $(21)$ & $(21)$ \\
\hline PF & 0 & 0 & 2 & 1 & 2 \\
(total: 7$)$ & $(4)$ & $(4)$ & $(6)$ & $(7)$ & $(7)$ \\
\hline FN & 0 & 0 & 6 & 6 & 6 \\
(total: 8$)$ & $(1)$ & $(0)$ & $(7)$ & $(8)$ & $(8)$ \\
\hline
\end{tabular}

Table 1. Correct classification results, possibilistic fusion

\begin{tabular}{|c|c|c|c|c|}
\hline \multirow{2}{*}{ Classified correctly, belief functions } & \multicolumn{3}{|c|}{ Sensors } & \multirow{2}{*}{ Fusion } \\
\cline { 2 - 4 } & IR & MD & GPR & 19 \\
\hline M & 10 & 9 & 13 & $(21)$ \\
\hline (total: 21$)$ & $(18)$ & $(9)$ & $(13)$ & 2 \\
PF & 3 & 0 & 1 & $(7)$ \\
\hline (total: 7) & $(4)$ & $(4)$ & $(6)$ & 6 \\
FN & 0 & 0 & 6 & $(8)$ \\
\hline
\end{tabular}

Table 2. Correct classification results, belief functions

The two decision rules, dec1 and $d e c 2$, give the same results for mines and friendly objects caused by clutter. In case of placed false alarms, 2 are correctly classified in case of dec 2 , which is a slight improvement with respect to dec1 and the same result as for the belief function fusion, shown in Table 2. It is not suprising that the placed false alarms are not so well detected by any of the methods, since our model is designed in order to favor the detection of mines. This is also the type of results expected from deminers. Regarding correct classification of mines, the results of the possibilistic fusion are slightly better than those obtained using the belief function method (19 mines detected, Table 2). This is due to the increased flexibility at the combination level. False alarms with no objects are correctly identified by the belief function method ( 6 out of 8 ), and it is the same result as for the two possibilistic decision rules. This result shows that a power of our methods is in decreasing the number of clutter-caused false alarms without decreasing the result of mine detection, thanks to knowledge inclusion.

All results have been obtained with the models proposed in Subsection 4.1, with the same parameters. Note that although the general shapes of the possibility distributions are important and have been designed based on prior knowledge, they do not need to be estimated very precisely, and the results are robust to small changes in these functions. What is important is that the functions are not crisp (no thresholding approach is used) and that the rank is preserved (e.g., an object with a measure value outside of the usual range should have a lower possibility degree than an object with a typical measure value). Two main reasons explain the experienced robustness: (i) these possibility distributions are used to model imprecise information, so they do not have to be precise themselves; (ii) each of them is combined in the fusion process (Subsection 4.2) with other pieces of information, which diminishes the importance and the influence of each of them.

Analysis regarding the robustness of the choice of the operator is also performed (within a class corresponding to the type of reasoning we want to achieve). Different operators within 
the same family have been tested, leading to the maximisation and minimisation of the possibility degrees of mines, thus being the safest and the least safe situations from the point of view of mine detection. The results obtained show that the model is robust indeed: all mines are detected in the second step, for all fusion schemes.

Differences between the results of Tables 1 and 2 can be formally explained as discussed in Subsection 4.3. For GPR, Eq. 53 explains why the results are the same for the two fusion approaches. In case of IR, Eq. 54 indicates that the possibilistic approach would favor mines more than the belief function approach, which is indeed the case here.

\section{Area Reduction: the SMART Approach}

\subsection{Overview of the Approach}

The aim of area reduction is to find which mine-suspected areas do not contain mines and this task is recognized as a mine action activity that should result in reduction in time and resources. Several well-known methods are in use to perform area reduction, especially using mechanical means. These expensive methods change and damage the environment and the ecosystem most of the time. To avoid this, some approaches have been developed that acquire the necessary information remotely, from air or space, using appropriate sensors associated with context information collected from the field and integrated in a geographical information system (GIS). The SMART project, funded by the European Commission/DG/INFSO, is among these approaches, and it is applied to Croatia. The goal of this project is to provide the human analyst with the SMART system, i.e. a GIS-based system augmented with dedicated tools and methods designed to use multispectral and radar data in order to assist in his interpretation of the possibly mined scene during the area reduction process. The usefulness of such image processing tools to help photointerpretation is, at first place, in the possibility to process automatically a large amount of data and help a visual analysis (SMART consortium, 2004). The use of SMART includes a field survey and an archive analysis in order to collect knowledge about the site, a satellite data collection, a flight campaign to record the data and the exploitation of the SMART tools by an operator to detect indicators of presence or absence of mine-suspected areas. With the help of a data fusion module based on belief functions and fuzzy sets, the operator prepares thematic maps synthesizing all the knowledge gathered with these indicators. These maps of indicators can be transformed into risk maps showing how dangerous an area may be according to the location of known indicators and into priority maps indicating which areas are designed to help the mined area reduction process. Preliminary results obtained using SMART have shown a reduction rate of $25 \%\left(0.98 \mathrm{~km}^{2}\right.$ over analyzed $\left.3.9 \mathrm{~km}^{2}\right)$ and an error rate of $0.1 \%$ for what SMART considers as not mined and is actually mined.

Fig. 1 illustrates the global SMART approach. This paper focuses on the fusion step, which provides an intermediary result in SMART, consisting of improved landcover classification maps, along with confidence values. Thus, it is a very useful result, exploited by the deminers together with the final result.

\subsection{Data and Their Specificities in SMART}

The available images include synthetic-aperture radar (SAR), multispectral, high resolution optical and satellite data. SAR data were collected with the E-SAR system of the German 
Aerospace Centre (DLR) in fully polarimetric P- and L-band and in vv-polarization (waves are vertically transmitted

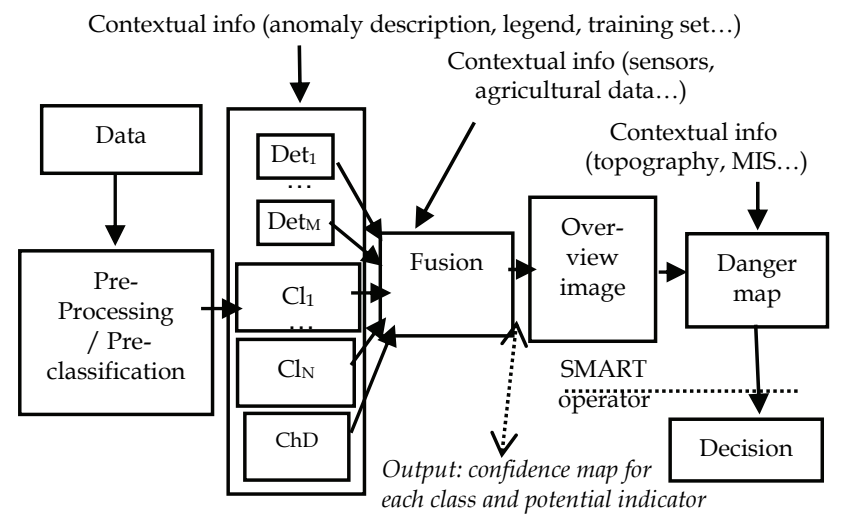

Fig. 1. The global SMART approach; Det - detector, $\mathrm{Cl}$ - classifier, $\mathrm{M}$ - number of detectors, $\mathrm{N}$ - number of classifiers, ChD - change detection, MIS - mine information system

and received) X-and C-band. Multispectral Daedalus data were collected with a spatial resolution of $1 \mathrm{~m}$ and in 12 channels, ranging from visible blue to thermal infrared. SAR and Daedalus data were geocoded. DLR also provided a complete set of RMK photographic aerial views recorded with a colored infrared film at a resolution of $3 \mathrm{~cm}$. This nongeocoded data set is used as evidence to control the processing tools and for qualitative interpretation by photo-interpreters. Finally, geocoded KVR-1000 black-and-white satellite images with a resolution of $2 \mathrm{~m}$, recorded before the war in Croatia, were purchased in order to assess the changes in the landscape due to the war.

The legend (expected classes in the images), derived based on the existing and gathered knowledge about the mined areas, is given in Table 3. Ground truth was provided as a set of regions (training regions and validation regions). In the fusion module, training regions are used for estimating the parameters of some of the proposed methods; validation regions are used for the evaluation of the results.

Table 4 summarizes the input of the fusion module.

A logistic regression classification was developed on SAR data at RMA (Borghys et al., 2004). The results consist of confidence images for each class, except for class 4 , which is not detected by this approach. A classification into hedges, trees, shadows, and rivers from SAR data has been developed at DLR (Keller et al., 2002). The method relies on the satisfaction of several criteria. The number of satisfied criteria directly provides the confidence images for hedges and trees (after scaling on [0,1]). Shadows and rivers, provided as binary images, are "discounted"(work done at RMA based on spectral characteristics of these types of landcover, and on existing landcover indices and meanings of Daedalus bands). Hedges and trees are then grouped to form class 6 using a maximum operator. Shadows and rivers are directly interpreted as classes 7 and 8 .

Several classifiers have been developed for Daedalus:

- a supervised classification method based on minimum distance has been developed at RMA and a decision image is provided (Keller et al., 2002); 
- a region-based classification was performed at Université Libre de Bruxelles (ULB) and confidence images interpreted as membership degrees to each class are provided;

- a belief function classification was developed at RMA and confidence images per class are provided (Keller et al., 2002).

\begin{tabular}{|l|l|}
\hline Class no. & Legend \\
\hline 1 & Abandoned agricultural land \\
\hline 2 & Agricultural land in use \\
\hline 3 & Asphalted roads \\
\hline 4 & Rangeland \\
\hline 5 & Residential areas \\
\hline 6 & Trees and shrubs \\
\hline 7 & Shadow \\
\hline 8 & Water \\
\hline
\end{tabular}

Table 3. Expected classes in the images

\begin{tabular}{|l|l|}
\hline Data type & Type of result \\
\hline SAR & Classification with confidence images per class \\
\hline SAR \& Daedalus & $\begin{array}{l}\text { Detection of hedges, trees, shadows, rivers, with confidence } \\
\text { degrees, sometimes discounted }\end{array}$ \\
\hline Daedalus & Supervised classification, result as a decision image \\
\hline Daedalus & Region-based classification with confidence images per class \\
\hline Daedalus & Belief function classification with confidence images per class \\
\hline SAR \& Daedalus & Binary detection of roads \\
\hline SAR & River detection (binary) \\
\hline Daedalus \& KVR & Change detection (binary image) \\
\hline
\end{tabular}

Table 4. Summary of the input of the fusion module

Road detection was performed at ULB and RMA. Linear structures are provided. They are dilated to obtain roads with a width corresponding to the real width.

A tool for river detection previously developed at Ecole Nationale Supérieure des Télécommunications ENST (Tupin et al., 1999) was used too. It is based on a Markovian approach. This is not directly a result of SMART but it is interesting to show how such knowledge can be introduced in the fusion process.

Change detection was obtained at ULB, based on a comparison between older KVR images and images made during the project. It provides mainly information on abandoned regions (class 1). Again, this is an important knowledge that both improves the landcover classification and provides interesting results for the construction of danger map.

Other anomaly detection and classification tools developed in SMART were not used either in the fusion module or at all. For example, detectors of power poles, hilltops and strategic locations are not included in the legend. Thus, they are not considered as input data for the fusion process, but they are added in the final results (construction of danger maps). 


\subsection{Fusion Strategies in SMART}

In all that follows, the computations are performed at pixel level. A final regularization step is then applied (see Section 5.4). Different fusion strategies have been developed (Bloch et al., 2007) and we present here three most promising ones.

Adding a global discounting factor (BF1): Here, we consider each classifier as one information source. The focal elements are the singletons and $\Theta$. The definition of $m(\Theta)$ takes into account both the fact that some classes are not detected (thus it should be equal to 1 at points where 0 is obtained for all detected classes) as well as global errors. We propose to use a discounting factor $\alpha$ equal to the sum of the diagonal elements of the confusion matrix, divided by the cardinality of the training areas. This discounting is applied on all masses defined as in the previous approach. Then:

$$
m(\Theta)=1-\alpha .
$$

Note that this uses explicitly the confidence matrix, which should be computed on the training areas for each classifier or detector. It results that at each step of the fusion, the focal elements are always singletons and $\Theta$. Decision rule can be maximum of belief, of mass or of pignistic probability (all being equivalent in this case).

This approach is very easy to implement and models in a simple way the fact that classifiers or detectors may not give any information on some classes and may be imperfect.

Use of confusion matrices for more specific discounting (BF2): Now each class of each classifier (or detector) is considered as a source and we take into account the behavior of the classifier with respect to the other classes, using the confusion matrices to define discounting for each class. From the confusion matrix computed from the decision made from one classifier and from training areas, we derive a kind of probability that the class is $C_{i}$ given that the classifier says $C_{j}$ as:

$$
c(i, j)=\frac{\operatorname{conf}(i, j)}{\sum_{i} \operatorname{con} f(i, j)},
$$

where the values conf $(i, j)$ denote the coefficients of the confusion matrix. We can ignore the low values and normalize the others, in order to reduce the number of non-zero coefficients (thus the number of focal elements in the following). We used a threshold value of 0.05 .

There are several ways to use this normalized confusion matrix, e.g. by setting $m\left(C_{i}\right)=c(i, j)$ for detected pixels in case of detectors and deriving a more complex method for classifiers. The most interesting way, applying to both classifiers and detectors in a similar way, is as follows. From $v\left(C_{j}\right)$ (denoting the result provided for class $C_{j}$ by a classifier), we define:

$$
m\left(C_{i}\right)=v\left(C_{j}\right) c(i, j)
$$

for all classes $C_{i}$ which are confused with $C_{j}$ (which provides $\sum_{i} m\left(C_{i}\right)=v\left(C_{j}\right)$ ), and:

$$
m(\Theta)=1-v\left(C_{j}\right) .
$$

In comparison to the simplest method, instead of keeping a mass on $C_{i}$ only (and $\Theta$ ), this mass is spread over all classes possibly confused with $C_{i}$, thus better exploiting the richness of the information provided by a classifier.

Fuzzy fusion (FUZZY): In order to compare the previous methods with a fuzzy approach, we test a simple method, where we choose for each class the best classifiers, and combine them with a maximum operator (possibly with some weights). Then decision is made according to a maximum rule. The choice is made based on the confusion matrix for each 
classifier or detector, by comparing the diagonal elements in all matrices for each class. In the illustrated example, the best detections, according to the confusion matrix of each classifier or detector are detailed in Subsection 5.5. They provide the inputs of the combination step, and a simple maximum operator performs well for this step.

This approach is very fast. It uses only a part of the information, which could also be a drawback if this part is not chosen appropriately. Some weights have to be tuned, which may need some user interaction in some cases. Although it may sound somewhat ad hoc, it is interesting to show what we can get by using the best parts of all classifiers.

\subsection{Knowledge Introduction and Spatial Regularization}

Knowledge inclusion is one of the main powers of our algorithms with respect to the commercial ones. This aspect has led to a lot of work in SMART, at different levels. Note that knowledge on the classifiers, their behaviors, etc. is already included in the previous steps. At this step, we use only the pieces of knowledge that directly provide information on the landcover classification. Other pieces of knowledge such as mine reports, etc. are not directly related to classes of interest, but rather to the dangerous areas, and are thus included in the danger map construction, which follows the fusion.

Several pieces of knowledge proved to be very useful at this step. They concern on the one hand some "sure" detection. Some detectors are available for roads and rivers, which provide areas or lines that surely belong to these classes. There is almost no confusion, but some parts can be missing. Then these detections can be imposed on the classification results. This is simply achieved by replacing the label of each pixel in the decision image by the label of the detected class if this pixel is actually detected. If not, its label is not changed. As for roads, additional knowledge is used, namely on the width of the roads (based on observations done during the field missions). Since the detectors provide only lines, these are dilated by the appropriate size, taking into account both the actual road width and the resolution of the images.

Another type of knowledge is very useful: the detection of changes between images taken during the project and KVR images obtained earlier. The results of the change detection processing provide mainly information about class 1 , since they exhibit the fields which were previously cultivated, and which are now abandoned. These results do not show all regions belonging to class 1 , but the detected areas surely belong to that class.

Then a similar process can be applied as for the previous detectors.

With the proposed methods, it was difficult to obtain good results on class 2, while preserving the results on class 1 that is crucial since it corresponds to fields no longer in use hence potentially dangerous. Therefore we use the best detection of class 2 (extracted from region based classification on Daedalus) as an additional source of knowledge.

As shown in Subsection 5.5, this additional knowledge introduction leads to better results.

The last step is regularization. Indeed, it is very unlikely that isolated pixels of one class can appear in another class. Several local filters were tested, such as a majority filter, a median filter, or morphological filters, applied on the decision image. A Markovian regularization approach on local neighborhoods was tested too. The results are not significantly better. A better approach is to use the segmentation into homogeneous regions provided by ULB. In each of these regions, a majority voting is performed: we count the number of pixels in this region that are assigned to each class and the class with the largest cardinality is chosen for the whole region (all pixels of this region are relabeled and assigned to this class). 
This type of regularization, which is performed at a regional level rather than at a local one, provides good results, as will be seen in the following.

\subsection{Results of BF1, BF2 and FUZZY}

Results shown here are obtained on the Glinska Poljana site in Croatia.

In case of BF1, for each classifier, the discounting factor $\alpha$ is calculated from the normalized sum of the diagonal elements of the confusion matrix obtained on the training areas (Table 5). After this type of fusion, a lot of confusion occurs between classes 1 and 2, but this is largely improved by knowledge inclusion, while noisy aspect is suppressed by regularization. In order to assess classification accuracy, we use user's accuracy (UA) and producer's accuracy (PA) measures that can be derived directly from confusion matrices. UA represents the probability that a given pixel will appear on the ground as it is classified. PA is the percentage of a given class that is correctly identified on the map. Table 6 shows some results for a few classes. Note that the most interesting classes for danger map building are 1, 2, 3 and 8 , and that, regarding the purpose of the project, PA is important for classes 1 and 8 , and UA for classes 2 and 3 .

\begin{tabular}{|l|l|l|l|}
\hline Team & Data type & Type of result & $\alpha$ \\
\hline RMA & SAR & $\begin{array}{l}\text { Classification with confidence images } \\
\text { per class (except class 4) }\end{array}$ & 0.41 \\
\hline DLR \& RMA & SAR \& Daedalus & $\begin{array}{l}\text { Detection of hedges, trees, shadows, } \\
\text { rivers, with confidence degrees for } \\
\text { hedges and trees; rivers and shadows } \\
\text { discounted based on Daedalus bands }\end{array}$ & 0.11 \\
\hline RMA & Daedalus & $\begin{array}{l}\text { Supervised classification, result as a } \\
\text { decision image }\end{array}$ & 0.46 \\
\hline ULB & Daedalus & $\begin{array}{l}\text { Region based classification with } \\
\text { confidence images per class }\end{array}$ & 0.80 \\
\hline RMA & Daedalus & $\begin{array}{l}\text { Belief function classification with } \\
\text { confidence images per class }\end{array}$ & 0.67 \\
\hline
\end{tabular}

Table 5. Discounting factors for method BF1

\begin{tabular}{|l|l|l|l|l|}
\hline Class & BC & BF1 & BF2 & FUZZY \\
\hline 1 (PA) & 0.84 & 0.81 & 0.78 & 0.89 \\
\hline 2 (UA) & 0.87 & 0.86 & 0.81 & 0.95 \\
\hline 3 (UA) & 0.88 & 0.96 & 0.96 & 0.98 \\
\hline 8 (PA) & 0.96 & 0.97 & 0.99 & 0.99 \\
\hline
\end{tabular}

Table 6. UA and PA for all three methods (after knowledge inclusion and spatial regularization) and the best classifier (BC) for each important class 
In addition, the "best classifier" (BC) in Table 6 is not always the same one, but the result is the one provided by the classifier that is the best for a particular class.

In order for the reader to have a better visual idea about the images containing the results, Fig. 2 contains the raw image of Glinska Poljana in a visible channel of Daedalus.

After classification of this area using BF1 (basic version), we obtain the results given in Fig. 3 (left), while knowledge inclusion and spatial regularization applied to these results lead to Fig. 3 (right). The color code in all classification results is as follows: class 1 - orange; 2 yellow; 3 - medium grey; 4 - light green; 5 - dark red; 6 - dark green; 7 - brown; 8 - blue.

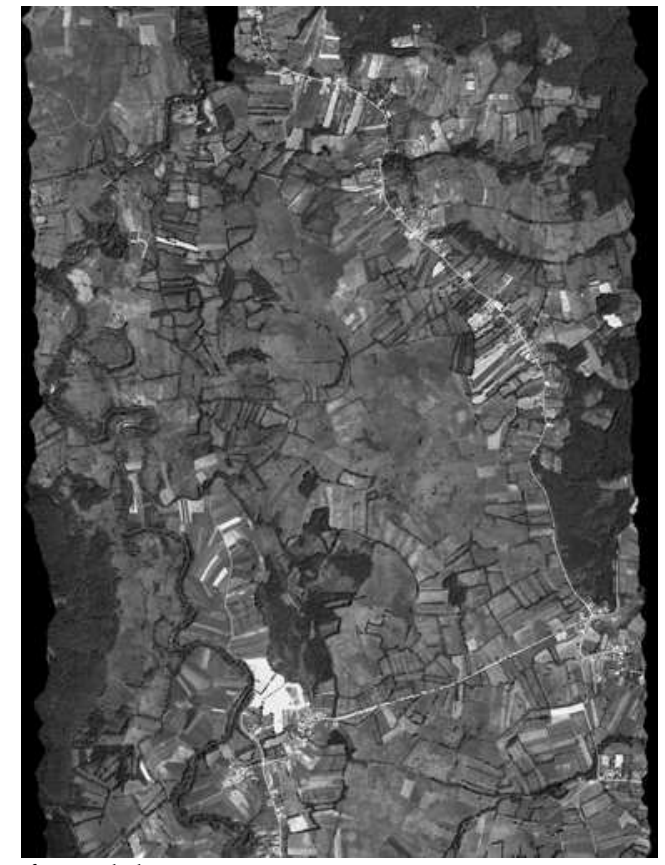

Fig. 2. Visible channel of Daedalus

The fusion module also provides confidence and stability images. The confidence image represents, at each pixel, the confidence degree of the decided class. The stability image is computed as the difference between the confidence in the decided class and confidence in the second most possible class. If the stability is high, this means that there is no doubt about the decision, and if it is low, the decision should be considered carefully. The confidence image and the stability image can be multiplied to provide a global image evaluating the quality of the classification in each point.

In the BF2 method, the confusion matrices for each classifier are normalized row by row, and the coefficients that are higher than 0.05 are used for discounting the corresponding classes. The results of the basic version of this type of fusion yield a poor detection of class 1 and a lot of confusion between this class and classes 2 and 7. In addition, class 4 is not detected and detection of class 3 is worse than with BF1. However, the results are largely improved by knowledge inclusion and confusions are strongly reduced. Finally, the noisy aspect is suppressed by the regularization, leading to an improved detection, in particular 
for class 8. Results are given in Fig. 4 left (after knowledge inclusion and spatial regularization). UA and PA are given in Table 6.

For the fuzzy method, the following outputs of classifiers have been used for each class:

1: SAR logistic regression, region-based classification, belief function classification and change detection;

2: region-based classification and belief function classification;

3: region-based classification and road detection;

4: region-based classification, minimum distance classification and belief function classification;

5: region-based classification and belief function classification;

6: region-based classification and SAR trees and hedges detection;

7: SAR logistic regression, SAR shadow detection, minimum distance classification and belief function classification; the maximum is discounted by a factor 0.5 , taking into account that this class is not really significant for further processing (shadows "hide" meaningful classes);

8: region-based classification, belief function classification and river detection.

The results of this fusion in its basic version are already very good, due to the fact that not all information provided by the classifiers is used, but only the best part of them. Further improvements are obtained by knowledge inclusion. After the regularization step, class 7 disappears, but this is not a problem since this class is not significant for further processing. Results of this method are shown in Fig. 4 right (after knowledge inclusion and spatial regularization). Table 6 contains PA and UA for this type of fusion too.
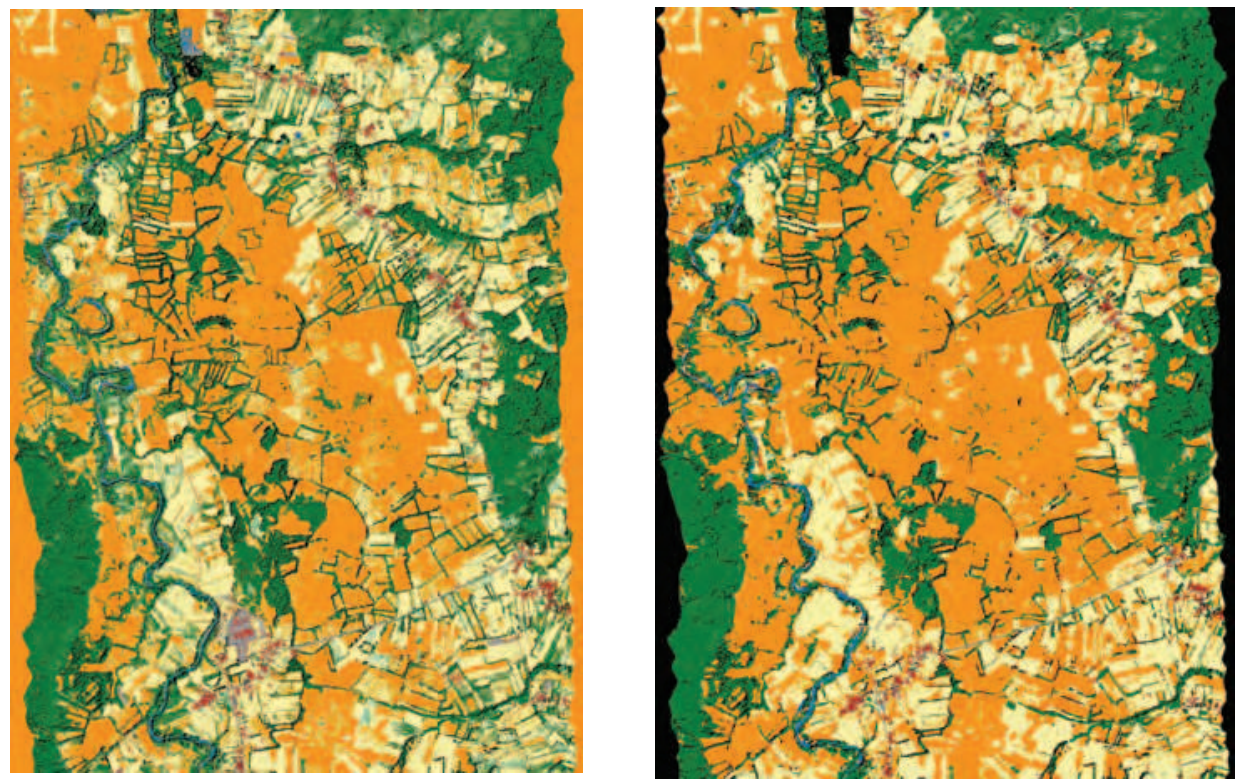

Fig. 3. BF1 results: basic (left), after knowledge inclusion and spatial regularization (right) 
In order to get a synthetic view of the results obtained by the three methods, the normalized sums of the diagonal elements of the confusion matrices are shown in Table 7. The two methods based on belief functions provide similar global results, BF1 being somewhat better. The differences appear mainly when looking individually at each class. The
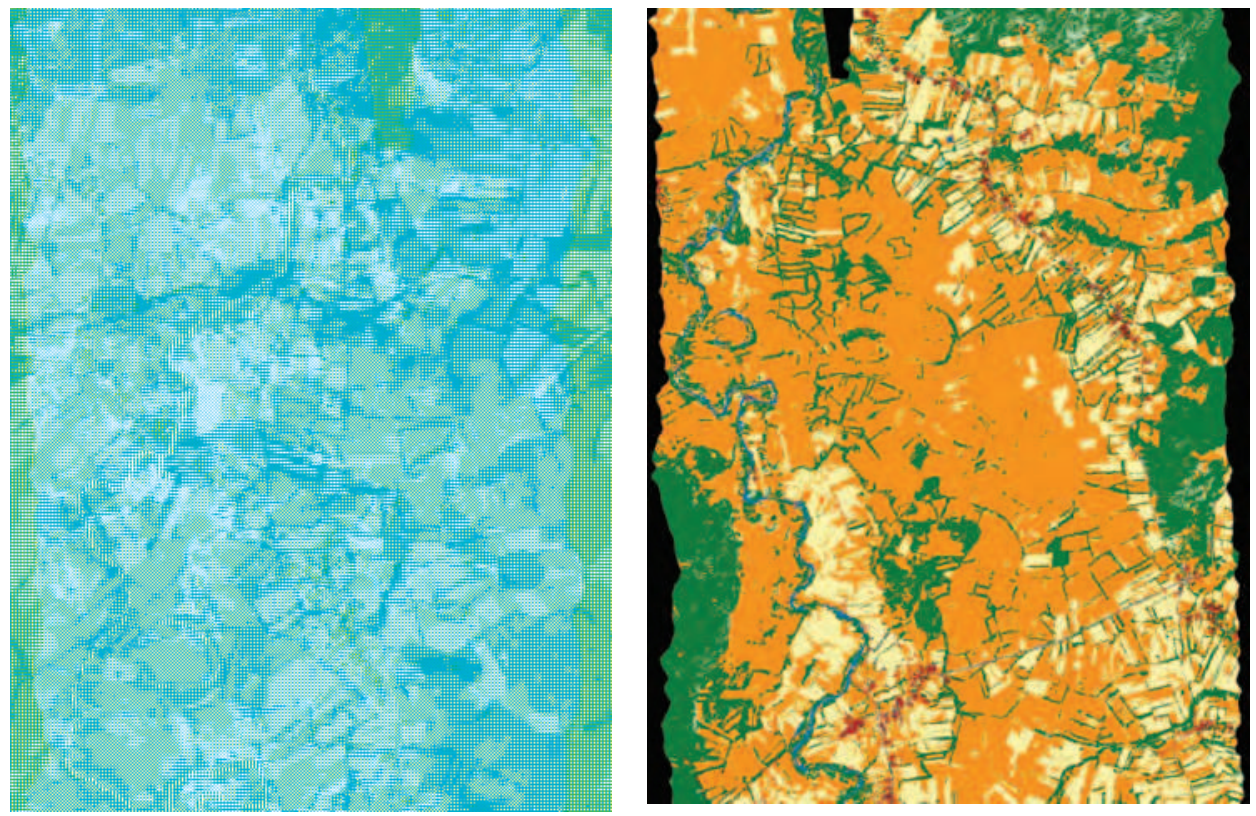

Fig. 4. Results with BF2 (left) and FUZZY (right), both after knowledge inclusion and spatial regularization

improvement achieved with knowledge inclusion is significant. Regularization provides an additional improvement. The final results are globally better than the ones obtained by each of the initial classifiers, as can be seen by comparing the values with those displayed in Table 5 (the best classifier provides a global accuracy of 0.80 ). The fuzzy method is the best in its basic version, since it already selects the best inputs, thus the improvement due to the next steps is not as important as for the belief function methods.

\begin{tabular}{|l|l|l|l|}
\hline Method & Basic & Knowledge inclusion & Spatial regularization \\
\hline BF1 & 0.70 & 0.81 & 0.85 \\
\hline BF2 & 0.65 & 0.78 & 0.81 \\
\hline Fuzzy & 0.79 & 0.83 & 0.84 \\
\hline
\end{tabular}

Table 7. Comparison of the normalized sum of diagonal elements of the confusion matrices for the three tested methods

\subsection{Danger Maps and First Results of SMART Validation}


The danger maps are synthetic documents designed to help the end users in their decisionmaking process regarding area reduction. They are created from results of all detection and classification tools and methods used in SMART (as well as some other sources such as fieldwork). These maps constitute the final output of the system and represent the basis for proposing areas for area reduction. Note that the results are for decision makers and that the reduction of a suspicious area is not an automatic process.

Four types of danger maps are developed in SMART (SMART consortium, 2004). The most useful continuous location maps, such as the one in Fig. 5, are obtained as a weighted sum of factors derived from the number of indicators of mine presence at each point (IMP), with a superimposition of vectors having a see-through inside, representing the number of indicators of mine absence (IMA) at each point.

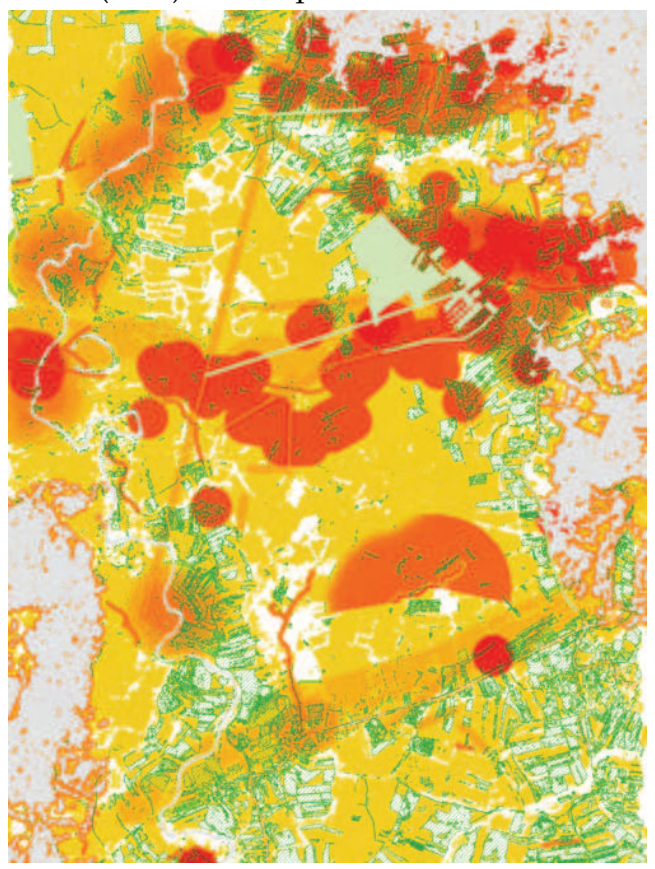

Fig. 5. Continuous location map (SMART consortium, 2004). Grey areas are outside of the scope of SMART, while no data exists for white areas. Demined areas are light green. IMAs are superimposed as parallel white and green lines. The degree of danger is on the scale from green (low) via yellow (intermediate) to red (high)

During the process of area reduction, the decision makers can refer to information relating to the IMA and the associated confidence values. The other key element is the information that concerns the IMP and the associated confidence values. As pointed out by the end users, this information can be of use for prioritizing the mine clearance operations.

Validation was done by blind tests at three sites in Croatia (Yvinec, 2005) having $3.9 \mathrm{~km}^{2}$ in total: Glinska Poljana $\left(0.63 \mathrm{~km}^{2}\right.$, fertile valley surrounded by hills), Pristeg $\left(1.5 \mathrm{~km}^{2}\right.$, rocky, Mediterranean area) and Čeretinci $\left(1.7 \mathrm{~km}^{2}\right.$, flat agricultural area). In each site clearing was 
performed after the flight campaign in order to have the true status of the mine presence, but this information was not available before the validation of produced danger maps. From these maps, a selection of areas proposed for area reduction was done, and areas considered as suspect were selected too. In average $25 \%$ of the mine-free area has been proposed for reduction: Glinska Poljana $-7.7 \%$, Pristeg $-9.0 \%$ and Čeretinci $-47 \%$. The error rate of $0.1 \%$ is relatively constant for all three sites. In addition to this technical evaluation, a panel of independent mine action experts working in Croatia has evaluated the SMART method and danger maps. They recognized SMART as a successful project that solved several crucial problems of the aerial survey of the suspected areas, especially by approved indicators of mine presence, efficient use of very different sensor techniques, data fusion and danger map functionalities. It has been found that it might be even more suited for risk assessment.

\section{Conclusion}

Several fusion methods for close range humanitarian mine detection and remote sensing mined area reduction are presented and compared. These methods are based on the belief functions as well as on the fuzzy/possibility theory.

Regarding close range mine detection, the differences at the combination step are mainly highlighted in this comparison. The modeling step is performed according to the semantics of each framework, but the designed functions are as similar as possible, so as to enhance the combination step. Different fusion operators are tested, depending on the information and its characteristics. An appropriate modeling of the data along with their combination in a possibilistic framework leads to a better differentiation between mines and friendly objects. The decision rule is designed to detect all mines, at the price of a few confusions with friendly objects. This is a requirement of this sensitive application domain (mines must not be missed). Still the number of false alarms remains limited in our results. The robustness of the choice of the operator is also tested, and all mines are detected for all fusion schemes. The proposed modeling is flexible enough to be easily adapted to the introduction of new pieces of information about the types of objects and their characteristics, as well as of new sensors.

As far as remote sensing mined area reduction is concerned, the concept of the whole method is described, developed within the SMART project, and most of the attention is given to the data fusion task. The proposed fusion approaches are to a large part original and constitute by themselves a result of the project. Results have been obtained on three test sites in Croatia, being representative of the South Eastern Europe, with the three most promising approaches, and as an example, fusion results for one of the sites are given here. Note that in order to apply the proposed methodology in another context, a new field campaign would be needed to derive and implement new general rules. We have shown how the results can be improved by introducing additional knowledge in the fusion process. A spatial regularization at a regional level further improves the results. At the end, the results are at least as good as the ones provided for each class by the best classifier for that class. Therefore they are globally better than any input classifier or detector. This shows the improvement brought by fusion.

The user has the possibility to be involved in the choice of the classifiers, in the choice of some of the parameters (in particular for the fuzzy fusion approach, some supervision is still 
required in the choice of the parameters), and the programs are flexible enough to allow him to modify them at wish.

The work done here is useful in many other applications, even in quite different domains, and constitutes thus a large set of methods and tools for both research and applicative work. The developed schemes have a noticeable variety and richness and constitute a real improvement over existing tools.

\section{Acknowledgement}

We thank the TNO Physics and Electronics Laboratory (The Hague, The Netherlands) for the permission to work on the data gathered on their test facilities within the Dutch HOM2000 project.

\section{References}

Acheroy, M. (2003). Mine Action Technologies: Problems and Recommendations, Journal for Mine Action, vol. 7, no. 3.

Acheroy, M. (2005). Spaceborne and Airborne Mined Area Reduction Tools, Workshop on Inventory and analysis of operationally validated results related to mine action space- and airborne surveys, Zagreb, Croatia, Scientific Council of CROMAC (invited paper).

Askelin, J-I. (1999). EOD IS -a Loaded Laptop. FOA magazine, Swedish Defense Research, No. 1.

Auephanwiriyakul, S.; Keller, J. M. \& Gader, P. D. (2002). Generalized Choquet fuzzy integral fusion. Information Fusion, Vol. 3, No. 1, pp. 69-85.

Bloch, I. (1996). Information combination operators for data fusion: a comparative review with classification. IEEE Trans. Systens, Man and Cybernetics, Vol. 26, pp. 52-67.

Bloch, I. \& Maître, H. (1997). Fusion of image information under imprecision, In: Series Studies in Fuzziness and Soft Computing, Aggregation and Fusion of Imperfect Information, Bouchon-Meunier, B. (Ed.), pp. 189-213, Springer, Berlin.

Bloch, I; Milisavljević, N. \& Acheroy, M. (2007). Multisensor Data Fusion for Spaceborne and Airborne Reduction of Mine Suspected Areas. Int'l journal of Advanced Robotic Systems, Vol. 4, No. 2, pp. 173-186.

Borghys, D.; Perneel, C.; Keller, M.; Pizurica, A. \& Philips, W. (2004). Supervised FeatureBased Classification of Multi-channel SAR Images Using Logistic Regression, Proc. of European Conference on Synthetic Aperture Radar (EUSAR), Vol. 2, pp. 949-952, Ulm, Germany.

Cremer, F.; Schutte, K.; Schavemaker, J. G. M. \& den Breejen, E. (2001). A comparison of decision-level sensor-fusion methods for anti-personnel landmine detection. Information Fusion, Vol. 3, No. 2, pp. 187-208.

de Jong, W.; Lensen, H.A. \& Janssen, Y.H.L. (1999). Sophisticated test facility to detect landmines, Proc. SPIE Conf. Detection and Remediation Technologies for Mines and Minelike Targets, Vol. 3710, pp. 1409-1418, Orlando, USA.

den Breejen, E.; Schutte, K. \& Cremer, F. (1999). Sensor fusion for anti personnel landmine detection, Proc. SPIE Conf. Detection and Remediation Technologies for Mines and Minelike Targets, Vol. 3710, pp. 1235-1245, Orlando, USA, April 1999. 
Delhay, S., Lacroix, V. \& Idrissa, M. (2005). PARADIS: GIS Tools for Humanitarian Demining. ISCRAM 2005: Information Systems for Crisis Response And Management Conference.

Denœux, T. (1995). A k-nearest Neighbor Classification Rule based on Dempster-Shafer Theory. IEEE Transactions on Systems, Man and Cybernetics, Vol. 25, No. 5, pp. 804813.

Dubois, D. \& Prade, H. (1980). Fuzzy sets and systems: Theory and applications, Collection: Mathematics in science and engineering 144, New York, London: Academic Press.

Dubois, D. \& Prade, H. (1985). A review of fuzzy set aggregation connectives. Information Sciences, Vol. 36, pp. 85-121.

Dubois, D. \& Prade, H. (1988). Representation and Combination of Uncertainty with Belief Functions and Possibility Measures. Computational Intelligence, Vol. 4, pp. 244-264.

Dubois, D.; Grabisch, M.; Prade, H. \& Smets, P. (2001). Using the transferable belief model and a qualitative possibility theory approach on an illustrative example: the assessment of the value of a candidate. Int'l Journal of Intelligent Systems, Vol. 16, No. 11, pp. 1245-1272.

Dubois, D. \& Prade, H. (2004). Possibilistic logic: a retrospective and prospective view. Fuzzy Sets and Systems, Vol. 144, pp. 3-23.

Duskunovic, I.; Stippel, G.; Pizurica, A.; Philips, W. \& Lemahieu, I. (2000). A New Restoration Method and its Application to Speckle Images, Proc. of the IEEE Int'l Conf. on Image Processing (ICIP 2000), pp. 273-276, Vancouver, Canada.

Gordon, J. \& Shortliffe, E. H. (1985). A Method for Managing Evidential Reasoning in a Hierarchical Hypothesis Space. Artificial Intelligence, Vol. 26, pp. 323-357.

IMSMA (1999). http:/ / www.imsma.org, Information Management System for Mine Action

International Campaign to Ban Landmines - ICBL (2005). Landmine Monitor 2005: Toward a Mine-Free World, Mines Action Canada, Handicap International, Human Rights Watch, Norwegian People's Aid.

JMU. http:/ / www.maic.jmu.edu, USA: website of the Mine Action Information Center at the James Madison University

Keller, M.; Milisavljević, N.; Suess, H. \& Acheroy, M. (2002). Reduction of Mine Suspected Areas by Multisensor Airborne Measurements: First Results, Proc. of SPIE Conference on Detection Technologies for Mines and Minelike Targets, Vol. 4742, pp. 857871, Orlando, USA.

Lee, R. H. \& Leahy, R. (1990). Multi-spectral Classification of MR Images Using Sensor Fusion Approaches, Proc. of SPIE Medical Imaging IV: Image Processing, Vol. 1233, pp. 149-157.

Le Hégarat-Mascle, S.; Bloch, I. \& Vidal-Madjar, D. (1998). Introduction of Neighborhood Information in Evidence Theory and Application to Data Fusion of Radar and Optical Images with Partial Cloud Cover. Pattern Recognition, Vol. 31, No. 11, pp.1811-1823.

Mascle, S.; Bloch, I. \& Vidal-Madjar, D. (1997). Application of Dempster-Shafer Evidence Theory to Unsupervised Classification in Multisource Remote Sensing. IEEE Transactions on Geoscience and Remote Sensing, Vol. 35, No. 4, pp. 1018-1031.

Milisavljević, N. \& Acheroy, M. (1999). An approach to the use of the Bayesian rule in decision level fusion for multisensor mine detection, Proc. Physics in Signal and Image Processing (PSIP), Paris, France, pp. 261-266. 
Milisavljević, N. \& Bloch, I. (2001). A Two-Level Approach for Modeling and Fusion of Humanitarian Mine Detection Sensors within the Belief Function Framework, Proc. of Applied Stochastic Models and Data Analysis, Vol. 2, pp. 743-748, Compiègne, France.

Milisavljević, N. \& Bloch, I. (2003). Sensor fusion in anti-personnel mine detection using a two-level belief function model. IEEE Trans. Systems, Man and Cybernetics, Part C, Vol. 33, No. 2, pp. 269-283.

Milisavljević, N.; Bloch, I.; van den Broek, S.P. \& Acheroy, M. (2003). Improving mine recognition through processing and Dempster-Shafer fusion of ground-penetrating radar data. Pattern Recognition, Vol. 36, No. 5, pp. 1233-1250.

Milisavljević, N. \& Bloch, I. (2005). Improving Mine Recognition through Processing and Dempster-Shafer Fusion of Multisensor Data. In: Computer-Aided Intelligent Recognition, Techniques and Applications, M. Sarfraz (Ed.), ch. 17, pp. 319-343, J. Wiley, New York, ISBN: 0-470-09414-1.

Perrin, S. (2001). Contribution à l'algorithmique multicapteur pour la détection de mines antipersonnel, Ph.D. dissertation, Ecole Centrale de Lille, USTL, France.

Shafer, G. (1976). A Mathematical Theory of Evidence, Princeton, NJ: Princeton Univ. Press.

SMART consortium (2004). SMART - final report.

Smets, P. (1990a). Constructing the pignistic probability fuction in a context of uncertainty, In: Uncertainty in Artificial Intelligence 5, Kanal, L. N.; Henrion, M.; Shachter, R. D. \& Lemmer, J. F. (Eds.), pp. 29-39, Elsevier, New York.

Smets, P. (1990b). The Combination of Evidence in the Transferable Belief Model. IEEE Transactions on Pattern Analysis and Machine Intelligence, Vol. 12, No. 5, pp. 447-458.

Smets, P. \& Kennes, R. (1994). The transferable belief model. Artificial Intelligence, Vol. 66, pp. 191-234.

Smets, P. (1994). What is Dempster-Shafer's model?, In: Advances in the Dempster-Shafer Theory of Evidence, Yager, R. R.; Fedrizzi M. \& Kacprzyk, J. (Eds.), pp. 5-34, Wiley, New York.

Stanley, R. J.; Gader, P. D. \& Ho, K. C. (2002). Feature and decision level sensor fusion of electromagnetic induction and ground penetrating radar sensors for landmine detection with hand-held units. Information Fusion, Vol. 3, No. 3, pp. 215-223.

Tupin, F.; Bloch, I. \& Maître, H. (1999). A First Step Towards Automatic Interpretation of SAR Images using Evidential Fusion of Several Structure Detectors. IEEE Transactions on Geoscience and Remote Sensing, Vol. 37, No. 3, pp. 1327-1343.

van Cleynenbreugel, I.; Osinga, S.A.; Fierens, F.; Suetens, P. \& Oosterlinck, A. (1991). Road Extraction from Multi-temporal Satellite Images by an Evidential Reasoning Approach. Pattern Recognition Letters, Vol. 12, pp. 371-380.

Yee, M.L. (2001). Multisensor probabilistic fusion for mine detection, Proc. SPIE Conf. Detection and Remediation Technologies for Mines and Minelike Targets, vol. 4394, pp. 959-969, Orlando, USA, April 2001.

Yvinec, Y. (2005). A Validated Method to Help Area Reduction in Mine Action with Remote Sensing Data, Proc. of the 4th Int'l Symposium on Image and Signal Processing and Analysis (ISPA 2005), Zagreb, Croatia.

Zadeh, L. (1965). Fuzzy sets. Information and Control, Vol. 8, No. 3, pp. 338-353. 


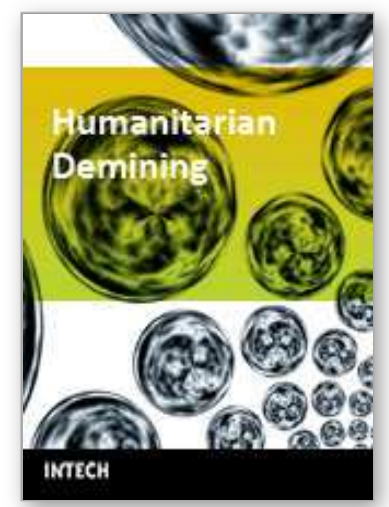

\author{
Humanitarian Demining \\ Edited by Maki K. Habib
}

ISBN 978-3-902613-11-0

Hard cover, 392 pages

Publisher I-Tech Education and Publishing

Published online 01, February, 2008

Published in print edition February, 2008

United Nation Department of Human Affairs (UNDHA) assesses that there are more than 100 million mines that are scattered across the world and pose significant hazards in more than 68 countries. The international Committee of the Red Cross (ICRC) estimates that the casualty rate from landmines currently exceeds 26,000 persons every year. It is estimated that more than 800 persons are killed and 1,200 maimed each month by landmines around the world. Humanitarian demining demands that all the landmines (especially AP mines) and ERW affecting the places where ordinary people live must be cleared, and their safety in areas that have been cleared must be guaranteed. Innovative solutions and technologies are required and hence this book is coming out to address and deal with the problems, difficulties, priorities, development of sensing and demining technologies and the technological and research challenges. This book reports on the state of the art research and development findings and results. The content of the book has been structured into three technical research sections with total of 16 chapters written by well recognized researchers in the field worldwide. The main topics of these three technical research sections are: Humanitarian Demining: the Technology and the Research Challenges (Chapters 1 and 2), Sensors and Detection Techniques for Humanitarian Demining (Chapters 3 to 8 ), and Robotics and Flexible Mechanisms for Humanitarian Demining respectively (Chapters 9 to 16$)$.

\title{
How to reference
}

In order to correctly reference this scholarly work, feel free to copy and paste the following:

Nada Milisavljevic, Isabelle Bloch and Marc Acheroy (2008). Multi-sensor Data Fusion Based on Belief Functions and Possibility Theory: Close Range Antipersonnel Mine Detection and Remote Sensing Mined Area Reduction, Humanitarian Demining, Maki K. Habib (Ed.), ISBN: 978-3-902613-11-0, InTech, Available from: http://www.intechopen.com/books/humanitarian_demining/multisensor_data_fusion_based_on_belief_functions_and_possibility_theory_close_range_antipersonnel

\section{INTECH}

open science | open minds

\section{InTech Europe}

University Campus STeP Ri

Slavka Krautzeka 83/A

51000 Rijeka, Croatia

Phone: +385 (51) 770447

\section{InTech China}

Unit 405, Office Block, Hotel Equatorial Shanghai

No.65, Yan An Road (West), Shanghai, 200040, China

中国上海市延安西路65号上海国际贵都大饭店办公楼 405 单元

Phone: +86-21-62489820 
Fax: +385 (51) 686166

Fax: +86-21-62489821

www.intechopen.com 
(C) 2008 The Author(s). Licensee IntechOpen. This chapter is distributed under the terms of the Creative Commons Attribution-NonCommercialShareAlike-3.0 License, which permits use, distribution and reproduction for non-commercial purposes, provided the original is properly cited and derivative works building on this content are distributed under the same license. 